\title{
Microbiome "state"
}

Beatriz García-jiménez, Mark D. Wilkinson

\section{Source}

Beatriz Garcí--liménez, Mark D. Wilkinson. (2019). Robust and automatic definition of

microbiome states.

A collection of constraints satisfied by the microbiota of one set of samples, that are not satisfied by other samples, allowing them to be reliably distinguished from one another. 